\title{
Wish you were here
}

\author{
Young scientists should use conference time to maximize exposure of and get feedback on their work \\ and to establish contacts within their scientific community.
}

\begin{abstract}
Most researchers attend conferences to share their most recent work, to gain new insights into their field and to build professional ties and collaborations (Nat. Chem. Biol. 1, $235,2005)$. Young investigators are, by definition, in the early stages of establishing their research focus and expertise, meaning conferences can be of particular importance as young scientists familiarize themselves with and make themselves known to the community. As the busiest conference season of the year begins, we offer a few thoughts for scientists young and old about how to make the most of meetings.

At any conference, being able to present your work in a poster session or in an oral presentation allows you to reach a different audience than you would encounter at your home institution. Be mindful of the likely perspective of those listening to your talk; if the conference is topically focused, your audience may already have an intimate knowledge of the field, and so you can focus on the more detailed aspects of your work.
\end{abstract} In contrast, if you are at a broadly defined meeting, you will want to present a larger amount of background information. The conference program and abstract book is a good first place to find out how your work fits in to the meeting as a whole as well as to get a head start in preparing your meeting itinerary.

If you are giving a talk, practice your entire talk well in advance of your presentation day to work out any delivery issues and to ensure that your talk fits within the allotted time. Ask colleagues to sit in on your talk to identify and resolve points of confusion or to help you anticipate what kinds of questions you might encounter. Consider organizing your talk so that you have a few short vignettes at the end of the presentation that can be included or bypassed as time allows. If you do find yourself over time during the presentation, skip to the end and summarize the major points instead of trying to get through the remainder of the slides more quickly; while the audience will undoubtedly be sympathetic to someone who didn't time their talk perfectly, egregious overages can be taken as disrespectful of the conference organizers and other speakers in the session.

Like giving a talk, presenting a poster requires sufficient planning, but it provides the advantage of getting to know your individual audience members and why they chose to visit your poster. When starting a conversation with someone new, keep in mind that you don't have to simply recite the contents of the poster; consider asking first about their background and what they want to learn, or set the stage for their questions with a short description of the work. Also keep in mind that posters are generally available for viewing outside of formal presentation times, so try to prepare your poster such that others can understand the basics in your absence.

Perhaps the most obvious benefit of presenting a poster is to get suggestions that will influence your work. Take the time as you prepare the poster to think about particular advice you hope to receive or what kinds of experts you would particularly like to hear from to take the next step in your research. Keep in mind that fresh perspectives can also lead to unexpected ideas or avenues of research, so be open to the idea of changing your research path or applying a new technique to your work.

Just as you value the input and feedback you get from other scientists on your work, be an active participant in the conference yourself. Pack in as many lectures, poster sessions and as much social time with scientists as you can. Seek to meet new people and 'switch off' as much as possible-don't check your phone or write emails during the sessions. Instead, try to use the time to actively learn and be inspired, and search for something to take away from each experience. For example, consider challenging yourself to formulate at least one question or tweet from each presentation (making sure to check the organizer's rules about reporting from the conference to confirm that tweeting, blogging or other sharing of information is allowed). Particularly at the interdisciplinary conferences typical of chemical biology, you may find new ways to think about your own work or new methods or molecules that might be useful from unexpected sources, while interactions with scientists outside of your immediate discipline may lead to fruitful collaborations. Besides the content of the lectures themselves, you can also use these sessions to sharpen your own presentation skills, noting styles or strategies that you appreciate.
Make the most of less obvious conference content as well. Larger society meetings often have sessions that are geared towards young scientists, offering career development tips, employment opportunities and workshops. At smaller conferences, there may also be overt opportunities for young scientists, such as discussion sessions with established scientists or oral sessions that are dedicated toward new researchers, but all of the social time is an opportunity to interact with people who may sooner or later be in a position to influence your career. Whether the format is more relaxed or more structured, you and your work will be noticed, so think about the impression you want to make on the people that you meet.

Some people you meet at conferences may be editors of scientific journals, including all of the Nature titles. At Nature Chemical Biology, we attend scientific conferences for many of the same reasons that active researchers do-to build and maintain relationships with our authors and referees and the community more broadly. Similarly, editors are often responsible for handling manuscripts in several diverse disciplines, so attending conferences is a good way to learn about new fields and to get the latest news on the fields we are more familiar with. This may include gaining a sense of the different models that exist within a research area and learning who favors one model over another, which proves useful in our editorial assessments of new manuscripts as well as informing our selection of referees for papers submitted to the journal. Editors are also interested in meeting the new members of a research area as well as identifying scientists asking intriguing or provocative questions about the research presented. We particularly view poster sessions as a way to discuss unpublished research that could potentially be appropriate for the journal.

Once you have decided to attend a conference, identify the goals that you wish to achieve and use your time accordingly. Keep an eye out for scientists whose work you're interested in and new ideas that might resolve sticking points in your own research, and engage with new friends, including journal editors. As we prepare for our own summer travel, we look forward to learning about your work and welcome questions about our editorial processes and criteria. 